\title{
El uso de diferentes poblaciones referenciales en el diagnóstico de los principales problemas nutricionales en niños y adolescentes
}

\author{
Different reference populations use in children and adolescents main nutritional \\ problems diagnosis
}

\author{
Jaime Pajuelo ${ }^{1,2}$, Mery Medrano ${ }^{3}$ \\ ${ }^{1}$ Instituto de Investigaciones Clínicas, Facultad de Medicina, Universidad Nacional Mayor de San Marcos. Lima, Perú. \\ ${ }^{2}$ Servicio de Endocrinología, Hospital Nacional Dos de Mayo. Lima, Perú. \\ ${ }^{3}$ Programa de Crecimiento y Desarrollo, Hospital Nacional Dos de Mayo. Lima, Perú.
}

\section{Resumen}

Introducción: La presencia de diferentes poblaciones de referencia utilizadas para diagnosticar el sobrepeso y la obesidad, en el grupo infantil, amerita hacer un análisis comparativo que permita sacar algunas conclusiones para su uso. Objetivos: Conocer las probables diferencias, en cuanto al diagnóstico nutricional se refiere, utilizando diferentes poblaciones referenciales. Diseño: Estudio descriptivo, comparativo y transversal. Lugar: Sección de Crecimiento y Desarrollo del Hospital Dos de Mayo y Centro Educativo Estatal de Lima Metropolitana. Participantes: Niños, de ambos géneros, comprendidos entre los 2 y 17 años. Intervenciones: Se estudió 219 niños, de ambos géneros, comprendidos entre los 2 a 5 años; y 1141 niñas y adolescentes de 9 a 17 años. En el primer grupo se hizo el diagnóstico de desnutrición crónica (talla/edad $<-2 \mathrm{DE}$ ) y obesidad (peso/talla $>+2 \mathrm{DE}$ ), de acuerdo a las referencias del Centro Nacional para Estadísticas en Salud (NCHS) 1983 y Organización Mundial de la Salud (OMS) 2005. En el segundo grupo, se utilizó el índice de masa corporal (IMC), para diagnosticar sobrepeso y obesidad en base a las referencias de Must, Cole y OMS, teniendo como criterios diagnósticos entre 85 a 95 y mayor del 95 percentil, para sobrepeso y obesidad, respectivamente. Principales medidas de resultados: Prevalencia de desnutrición crónica, sobrepeso y obesidad. Resultados: En los niños de 2 a 5 años, y para la desnutrición crónica, se ha encontrado una prevalencia de 1,8\% (NCHS) y 5,9\% (OMS) y, para la obesidad, 4,6\% (NCHS) y $9,1 \%$ (OMS), respectivamente. En el grupo de 9 a 17 años, por encima del 85p, la prevalencia fue $30,3 \%$ (Must), 33\% (Cole) y 35,2\% (OMS), respectivamente; en cuanto al sobrepeso, $21 \%$ (Must), 26,3\% (Cole) y $18 \%$ (OMS); y para la obesidad 9,3\% (Must), 6,7\% (Cole) y 17,2\% (OMS), respectivamente. Conclusiones: Los resultados son diferentes en función de la referencia que se utilice. La referencia de la OMS identifica mayor prevalencia de desnutrición crónica y de obesidad en el grupo de preescolares. Lo mismo sucede para el grupo de niñas y adolescentes, en lo que se refiere al sobrepeso y obesidad. Todas las diferencias encontradas responden a un criterio estadístico más que a un criterio biológico.

Palabras clave: Desnutrición infantil; sobrepeso; obesidad; evaluación nutricional; grupos poblacionales.

\begin{abstract}
Introduction: The existence of different reference populations for overweight and obesity diagnosis in children warrants comparative analysis. Objectives: To determine existing differences in nutritional diagnosis using different reference populations. Design: Descriptive, comparative and transversal study. Setting: Hospital Dos de Mayo Growth and Development Section and a Lima Metropolitan State School. Participants: Male and females children aged between 2 and 17 years. Interventions: Two hundred and nineteen children 2 to 5 year-old were studied, as well as 1141 girls and adolescents between 9 to 17 years. In the first group chronic malnutrition (height / age < -2 SD) and obesity (weight/ height $>+2$ SD) diagnosis was done according to the 1983 National Center for Health Statistics (NCHS) and 2005 World Health Organization (WHO) references. In the second group body mass index (BMI) was used for overweight and obesity diagnosis, based on Must, Cole and WHO references, and using between 85 and 95 and greater than 95 percentile for overweight and obesity diagnosis criteria. Main outcome measures: Prevalence of chronic malnutrition, overweight and obesity. Results: In children between 2 and 5 years, chronic malnutrition prevalence was respectively 1,8\% (NCSH) and 5,9\% (WHO), and 4,6\% (NCSH) and $9,1 \%$ (WHO) for obesity. In the 9 to 17 years group, the above $85 p$ prevalence was 30,3\% (Must), 33\% (Cole) and 35,2\% (WHO); for overweight, respectively $21 \%$ (Must), 26,3\% (Cole) and 18\% (WHO), and for obesity 9,3\% (Must), $6,7 \%$ (Cole) and 17,2\% (WHO). Conclusions: The results differ depending on the reference used. The WHO reference identifies higher prevalence of chronic malnutrition and obesity in the preschool group. The same applies to the group of girls and adolescents in regard to overweight and obesity. All differences found reflect a statistical approach rather than a biological criterion.
\end{abstract}

Key words: Child nutrition disorders; overweight; obesity; nutrition assessment; population groups.

\section{INTRODUCCIÓN}

Los problemas nutricionales de mayor magnitud, que son identificados mediante la antropometría, son la desnutrición crónica y la obesidad en los preescolares, y el sobrepeso y la obesidad en el grupo de niños y adolescentes.

A diferencia de lo que sucede en el adulto, donde existe un denominador común en el uso de las referencias para el diagnóstico ${ }^{(1)}$, en los grupos del preescolar, niños y adolescentes no se dan estas condiciones debido a que llevaría a diagnósticos en función de la referencia utilizada, lo que traería aparejado conclusiones que en vez de aclarar el problema lo confundirían.

Históricamente, la primera información que se tuvo en forma regular y sistemática del uso de la antropometría en el crecimiento lineal data de la segunda mitad del siglo XVIII, cuando Philibert de Montheillard midió la talla de su hijo, a intervalos regulares, hasta los 18 años de edad.

En el siglo XX, se utilizó mucho la referencia de Gómez para diagnosticar desnutrición global, en base a la relación peso/edad (2); esta referencia fue muy usada en los ámbitos hospitalarios. Pos- 
teriormente, se incorporó los indicadores talla/edad (desnutrición crónica) y peso/ talla (desnutrición aguda).

A partir de los años 80 , empiezan a estructurarse tablas en base a estudios poblacionales que permitiesen servir como referencias internacionales dirigidas al grupo de 0 a 18 años, y no solamente intrahospitalarias sino en estudios de campo. Es así que en EE UU, en base a dos poblaciones infantiles diferentes, 0 a 36 meses en los estudios del Fels Research Institute, Yellow Spring, Ohio y de 2 a 18 años del National Center for Health Statistisc (NCHS), se genera el patrón referencial utilizado internacionalmente y dado a conocer el año $1983^{(1)}$. Esta referencia permitió su uso para el análisis de los tres indicadores en los preescolares (peso/edad, talla/edad y peso/talla). Un grupo de asesores de la Organización Mundial de la Salud (OMS) consideró que los datos recogidos por el NCHS eran los más aptos para ser utilizados como población de referencia internacional ${ }^{(3)}$.

Posteriormente, y ante la recomendación del uso del índice de masa corporal (IMC) como instrumento de diagnóstico, en el año 1995, un grupo de expertos de la Organización Mundial de la Salud (WHO) ${ }^{(4)}$ recomendó el uso de la clasificación percentilar de Must ${ }^{(5)}$, a partir de los 6 a los 18 años. Esta clasificación guarda una estrecha relación con la mencionada anteriormente, por cuanto se hizo en base a la información de los mismos niños, o sea del First National Health and Nutrition Examination Survey (NHANES I).

Otra población de referencia que emerge alrededor del año 2000 es la propuesta por el CDC-NCHS en base a 9 estudios transversales realizados entre los años 1970 y 1994, en diferentes estados de los EE UU. La muestra se hizo en base a niños y adolescentes comprendidos entre los 5 a 17 años ${ }^{(6)}$.

En el año 2000, la Comisión Internacional sobre la Obesidad (IOTF) recomienda el uso de la clasificación percentilar de Cole ${ }^{(7)}$, que presenta valores desde los 2 hasta los 18 años y que reúne una serie de encuestas representativas de varios países, como Brasil, Rusia, Estados Unidos, Inglaterra, Hong Kong, Holanda y Singapur. En esta referencia, los valores correspondientes al 85 y 95 percentil presentan una correspondencia con los IMC de 25 y 30, utilizados en adultos para definir sobrepeso y obesidad, respectivamente.

Pasados los años, la OMS consideró que la referencia del NCHS era muy antigua y que era menester contar con una referencia actualizada, tomando en cuenta algunas mejoras en cuanto a la calidad de la selección de los niños que participarían en el estudio. Eso se manifestó en los requisitos que era necesario cumplir para su participación. Estos requisitos fueron: ausencia de problemas sanitarios, ambientales o económicos que interfirieran el crecimiento; disposición de la madre para cumplir las recomendaciones alimenticias, nacimiento a término (37 a 42 semanas), nacimiento de un solo hijo, ausencia de enfermedades significativas, haber tenido lactancia materna exclusiva hasta los 4 meses de edad y que sus madres no fueran fumadoras. Por otro lado, estos niños no solo deberían ser de los Estados Unidos, sino de otros países; así se incorporaron los de Noruega, Ghana, Omán, India y Brasil. Las tablas fueron presentadas con las clásicas relaciones del peso/edad, talla/edad y peso/talla para menores de 5 años. Adicionalmente, incorporaron la relación IMC/edad, hasta los 18 años ${ }^{(8)}$.

El objetivo del presente estudio fue describir las diferencias que puedan existir, en cuanto al diagnóstico de desnutrición se refiere, utilizando diferentes patrones referenciales. Con esta finalidad se ha utilizado dos estudios: uno con niños de $\operatorname{los} 2$ a 5 años, en quienes se ha utilizado las clasificaciones de la $\mathrm{OMS}{ }^{(2,8)}$, y el otro con adolescentes de 9 a 17 años, donde se ha usado las clasificaciones de Must ${ }^{(2)}$, Cole ${ }^{(7)}$ y la última de la OMS ${ }^{(8)}$.

\section{MÉTODOS}

En primer lugar, se realizó un estudio descriptivo, donde participaron 219 niños, comprendidos desde los 2 hasta los 5 años, $52 \%$ de sexo femenino y $48 \%$ de sexo masculino. A todos se los pesó y talló siguiendo las recomendaciones internacionalmente aceptadas ${ }^{(9)}$.
Para los fines del diagnóstico, se relacionó la talla/edad y el peso/talla, con el objetivo de identificar a los preescolares con desnutrición crónica y obesidad. Los criterios diagnósticos utilizados fueron: menor de menos 2 del desvío estándar, para la desnutrición crónica (talla/edad), y mayor de más dos desvíos estándar de la relación peso/talla para la obesidad. Uno de los patrones de referencia que se usó fue el del Centro Nacional para Estadísticas en Salud (NCHS), promovido por la Organización Mundial de la Salud (OMS) ${ }^{(2)}$ y utilizado hasta la actualidad, y el segundo es el que corresponde y el que recomienda la OMS desde el año $2005^{(8)}$.

La tabla 1 permite observar los valores de talla para la edad, que corresponden al menos dos desvío estándar de la curva de Gauss, de acuerdo a las referencias del NCSH y de la OMS. Se ha elegido al grupo de 2 y 4 años como ejemplo de lo que se pretende demostrar. Internacionalmente, se acepta que la relación talla/ edad sirve para diagnosticar desnutrición crónica, siempre y cuando esta relación esté por debajo o sea menor que el menos dos desvío estándar. En la tabla se observa la clara diferencia que existe entre las dos referencias mencionadas.

La tabla 2 muestra los valores correspondientes al más dos desvío estándar de la relación talla/peso de las referencias del NCHS y OMS. Esta relación permite identificar a los niños obesos, siempre y cuando se encuentre por encima del valor en mención. Para fines del ejemplo, solo se muestra las tallas de 86 a $90 \mathrm{~cm}$ y de 100 a $104 \mathrm{~cm}$. En ese sentido, se puede ver que los valores de la OMS son menores que la del NCHS y por ende tienen más posibilidad de identificar mayor cantidad de niños con obesidad.

En el segundo estudio, fueron 1141 niñas, comprendidas entre los 9 y 17 años. A todas se las pesó y talló, siguiendo la metodología internacionalmente aceptada ${ }^{(9)}$. Con ambas medidas, se calculó el $\operatorname{IMC}\left(\mathrm{kg} / \mathrm{m}^{2}\right)$.

El diagnóstico nutricional se hizo con el sistema percentilar, tomando como criterios diagnósticos para el sobrepeso valores que se encontraban entre 85 y 95 y para la obesidad mayor del 95 percentilo. Se hizo uso de tres tablas percentilares: 
Tabla 1. Valores de talla/edad correspondientes al menos dos desvío estándar, de acuerdo a población de referencia según edad por años y meses.

\begin{tabular}{cccccc}
\hline \multicolumn{3}{c}{ Edad } & $\begin{array}{c}\text { NCHS } \\
<-2 \text { OMS }\end{array}$ & Edad & \multicolumn{2}{c}{ NCHS } & $\begin{array}{c}\text { OMS } \\
<-2 \text { DE }\end{array}$ \\
\hline 2 & 79,2 & 81 & 4 & 94,4 & 94,9 \\
2,01 & 79,9 & 81,7 & 4,01 & 95 & 95,4 \\
2,02 & 80,6 & 82,5 & 4,02 & 95,5 & 95,9 \\
2,03 & 81,3 & 83,1 & 4,03 & 96,1 & 96,4 \\
2,04 & 82 & 83,8 & 4,04 & 96,6 & 96,9 \\
2,05 & 82,7 & 84,5 & 4,05 & 97,1 & 97,4 \\
2,06 & 83,4 & 85,1 & 4,06 & 97,7 & 97,8 \\
2,07 & 84,1 & 85,7 & 4,07 & 98,2 & 98,3 \\
2,08 & 84,7 & 86,4 & 4,08 & 98,7 & 98,8 \\
2,09 & 85,4 & 86,9 & 4,09 & 99,2 & 99,3 \\
2,1 & 86 & 87,5 & 4,1 & 99,7 & 99,7 \\
2,11 & 86,7 & 88,1 & 4,11 & 100,2 & 100,2 \\
\hline
\end{tabular}

NCHS = National Center for Health Statistisc

OMS = Organización Mundial de la Salud.

$\mathrm{DE}=$ desviación estándar.

la de Must y col (1991), la de Cole y col (2000) y la de la Organización Mundial de la Salud (OMS) (2005).

En la tabla 3, se observa la distancia que existe entre el valor correspondiente al 85 y 95 percentil de IMC, de cada población de referencia, por grupos de edad. Para las tres poblaciones, los valores que se encuentran dentro de estos percentiles tienen sobrepeso. Claramente se observa que la de Cole, indistintamente de la edad, presenta una distancia mayor, lo que en otras palabras significa que hay mayor probabilidad de que un niño u adolescente se encuentre dentro de este rango. Para el ejemplo, solo se muestra tres edades, pero la tendencia es la misma para las otras edades.

\section{RESULTADOS}

El figura 1 muestra la prevalencia de la desnutrición crónica y de obesidad en niños de 2 a 5 años, de acuerdo a diferentes referencias. Utilizando el patrón referencial de la OMS, se identifica mayor cantidad de niños con desnutrición crónica $(4,6 \%)$ que el del NCHS (1,8\%). Lo mismo ocurre con el diagnóstico de obesidad, siendo la de la OMS que mayor prevalencia da, 9,1\%, mientras la NCHS alcanza solamente 5,9\%. Existe una

Tabla 2. Valores de peso/talla correspondientes al más dos desvío estándar, de acuerdo a población de referencia.

\begin{tabular}{|c|c|c|c|c|c|}
\hline \multirow[b]{2}{*}{ Talla (cm) } & $\mathrm{NCHS}$ & OMS & \multirow[b]{2}{*}{ Talla (cm) } & NCHS & OMS \\
\hline & \multicolumn{2}{|c|}{$>+2 \mathrm{DE}$} & & \multicolumn{2}{|c|}{$>+2 \mathrm{DE}$} \\
\hline 86 & 15,3 & 14,1 & 100 & 18,8 & 18,2 \\
\hline 86,5 & 15,4 & 14,2 & 100,5 & 18,9 & 18,4 \\
\hline 87 & 15,5 & 14,4 & 101 & 19,1 & 18,5 \\
\hline 87,5 & 15,6 & 14,5 & 101,5 & 19,2 & 18,7 \\
\hline 88 & 15,7 & 14,7 & 102 & 19,4 & 18 \\
\hline 88,5 & 15,8 & 14,8 & 102,5 & 19,5 & 19,1 \\
\hline 89 & 16 & 14,9 & 103 & 19,7 & 19,3 \\
\hline 89,5 & 16,1 & 15,1 & 103,5 & 19,9 & 19,5 \\
\hline 90 & 16,2 & 15,2 & 104 & 20 & 19,7 \\
\hline
\end{tabular}

NCHS = National Center for Health Statistisc.

OMS = Organización Mundial de la Salud.

$\mathrm{DE}=$ desviación estándar.

diferencia en la identificación de los dos grandes problemas nutricionales en este grupo de edad.

La figura 2 permite observar la prevalencia de sobrepeso y obesidad en niñas de 9 a 17 años, con las diferentes referencias utilizadas. Para este grupo de edad, se ha trabajado con las referencias de Must ${ }^{(1)}$, Cole ${ }^{(5)}$ y la de la OMS ${ }^{(6)}$. Se muestra la prevalencia en conjunto sin hacer disquisición entre el sobrepeso y la obesidad. En este sentido, lo primero que se puede apreciar es que las prevalencias son diferentes, siendo la de la OMS la que identifica el mayor porcentaje de niños con estas patologías $(35,2 \%)$; por el contrario, la de Must da el menor porcentaje $(30,3 \%)$.

Al desagregar el cuadro anterior en sus componentes, que son el sobrepeso y la obesidad, se observa que la que mayor identifica el sobrepeso es la de Cole (26,3\%), le sigue la de Must (21\%) y por último la de OMS (18\%). En lo que respecta a la obesidad, la figura es diferente; el primer lugar lo ocupa la de la OMS $(17,2 \%)$, seguida de la de Must $(9,3 \%)$ y finalmente la de Cole (6,7\%) (figura 3).

\section{DISCUSIÓN}

Permanentemente ha existido el interés y la preocupación de los países en el estudio nutricional del grupo preescolar, ya que siempre este ha sido considerado como el más vulnerable para los problemas nutricionales. Para el diagnóstico de estos problemas se ha utilizado la población de referencia del NCHS ${ }^{(2)}$; pero, a partir del año 2005, irrumpe la referencia de la $\mathrm{OMS}^{(8)}$, lo que de alguna manera podría generar problemas cuando se comparen estudios con la finalidad de poder decir si la situación ha mejorado o no.

En el caso del presente estudio y para la desnutrición crónica o retardo de crecimiento, en función de las referencias utilizadas, los resultados son diferentes: para el NCHS $1,8 \%$ y para la OMS 4,6\%. La explicación que se le puede dar a estas diferencias radica, desde nuestro punto de vista, en los valores de la mediana, pero fundamentalmente al que corresponde al menos dos desvío estándar de cada referencia. 
Tabla 3. Distancia que existe entre los valores correspondientes al 85 y 95 percentil de cada población de referencia, según edad en años.

\begin{tabular}{cccc}
\hline Edad & Must $^{(1)}$ & Cole $^{(5)}$ & OMS \\
\hline 9 & 2,6 & 3,7 & 1,8 \\
12 & 3,8 & 5 & 2,4 \\
15 & 4,1 & 5,2 & 2,6 \\
\hline
\end{tabular}

OMS = Organización Mundial de la Salud.

Internacionalmente, se reconoce que todos los niños cuya talla se encuentren por debajo del menor de menos dos desvío estándar son identificados como desnutridos crónicos; es por esta razón que existe más probabilidad en la referencia que tiene un valor mayor del menos dos desvío estándar que la otra. Por ejemplo, en un niño de 2 años exactos, el valor del menos dos desvío estándar es 79,2 cm (NCHS) y 81 cm (OMS). Los niños que tuvieran una talla que va de $79,3 \mathrm{~cm}$ a $80 \mathrm{~cm}$ no son desnutridos crónicos para la NCHS, pero sí para la OMS.

El otro problema que afronta este grupo de edad es la obesidad, cuya tendencia epidemiológica es cada vez a incrementarse ${ }^{(10-12)}$. Su diagnóstico se hace mediante la relación peso/talla y su criterio diagnóstico para identificar obesos es todos aquellos que se encuentran por encima de más de dos desvío estándar. En ese sentido, el NCHS ha identificado a $5,9 \%$ y la OMS a 9,1\%. Acá, la explicación es la misma y, como ejemplo, para un niño que mide $86 \mathrm{~cm}$, el valor del más dos desvío estándar es $15,3 \mathrm{~kg}$ (NCSH) y 14,1 kg (OMS), lo cual refleja que las prevalencias van a ser siempre diferentes, a predomino de la de la OMS. Un estudio comparó la prevalencia de obesidad utilizando la relación peso/talla (NCHS) y el IMC (Cole), en niños de 2 a 5 años, dando mayores prevalencias la de Cole ${ }^{(13)}$.

En el grupo de niños y adolescentes, el problema de mayor magnitud es el sobrepeso y la obesidad. La explicación de las diferencias encontradas en los niños y adolescentes de 9 a 17 años, también podrían explicarse desde el punto de vista estadístico. Revisando los valores que corresponden al 85 y 95 percentil de

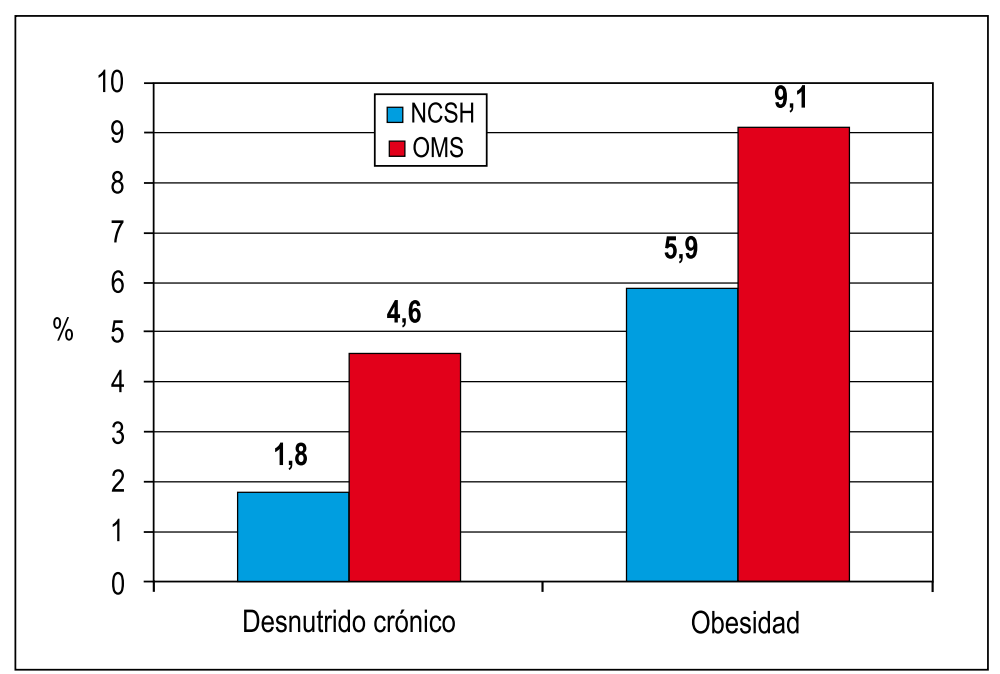

Figura 1. Prevalencia de desnutrición crónica y de obesidad en niños de 2 a 5 años. cada población de referencia utilizado, se observa que la distancia que separan estos percentiles, para el caso de los niños de 9 años, es menor para la de la OMS ${ }^{(1,8)}$, le sigue la de Must ${ }^{(2,6)}$ y por último la de Cole ${ }^{(3,7)}$. Esta misma tendencia se encuentra para los otros grupos de edad. En este sentido, es lógico encontrar más personas con sobrepeso con la de Cole (26,3\%), luego la de Must (21\%) y finalmente la de la OMS (18\%). Lo contrario sucede con la obesidad, ya que el espacio que queda por encima del 95 percentilo es menor en la de Cole (6,7\%), luego la de Must (9,3\%) y finalmente la de la OMS $(17,2 \%)$.
Estos detalles estadísticos tienen una gran repercusión, si es que no son tomados en cuenta. Cuando se utiliza la antropometría, para el caso de comparar estudios, es menester y obligatorio que esa comparación se haga siempre y cuando se use el mismo indicador de evaluación y los mismos elementos que se utilizó para el análisis de esa información. Los elementos en mención son la población de referencia, el sistema de clasificación y los criterios diagnósticos. En el caso de los grupos de este estudio, lo único que no es igual son las poblaciones de referencia y esa es la razón de las diferencias.

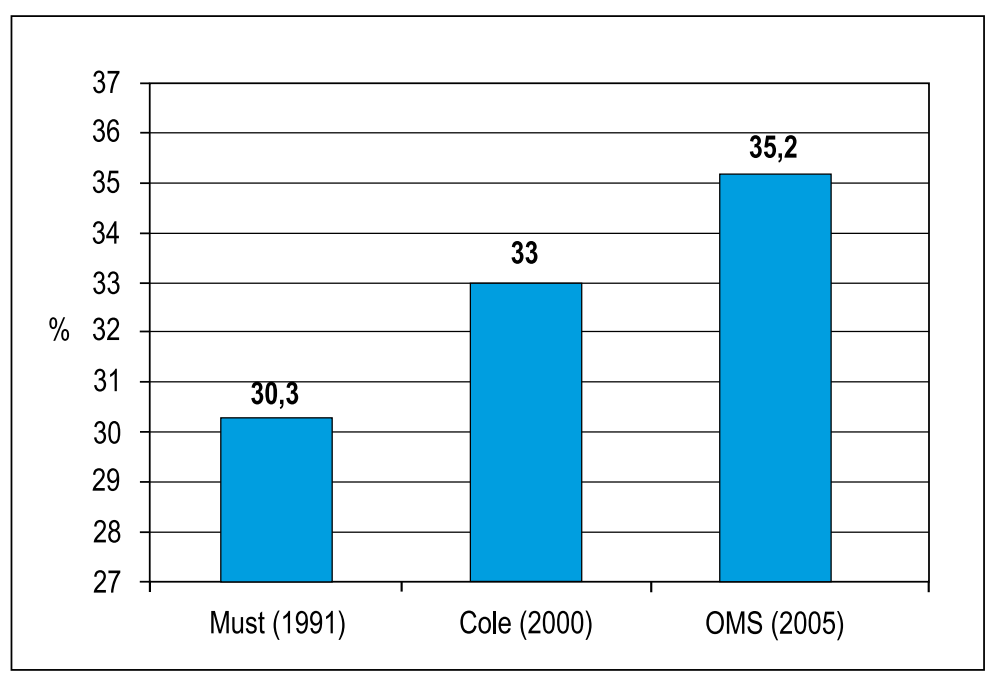

Figura 2. Prevalencia de sobrepeso y obesidaden niñas de 9 a 17 años, de acuerdo a diversas poblaciones referenciales. 


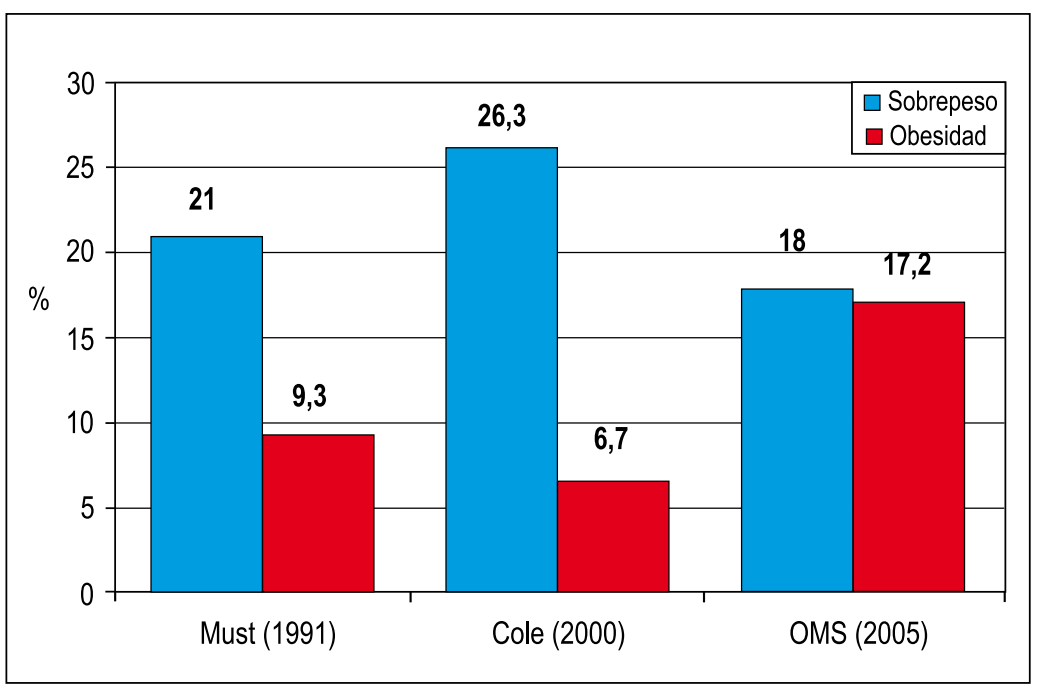

Figura 3. Prevalencia de sobrepeso y obesidad al desagregar los componentes de las poblaciones referenciales.

En el Perú, la mayoría de estudios referidos al sobrepeso y obesidad en niños y adolescentes han sido analizados con la población de referencia de Must (14). Actualmente, tomando en cuenta la recomendación de la OMS, los trabajos empezarán a hacerse con este patrón y si hipotéticamente comparamos estudios de hoy con los de ayer se va a concluir que ha disminuido la prevalencia de sobrepeso y aumentado la prevalencia de obesidad, cosa que no es real sino producto de un cambio en la población referencial.

La mayoría de trabajos relacionados al tema han comparado las referencias de Must con la de Cole y algunos con la CDC. El trabajo de Wang ${ }^{(15)}$, uno de los más extensos que abarcó niños de 6 a 18 años, de Estados Unidos (6 108), Rusia (6 883) y China (3 014), comparó las referencias de Must y Cole. Señaló que, al comparar el conjunto de sobrepeso y obesidad, la de Cole identificó mayores prevalencias que la de Must. En el caso específico de la obesidad, la de Must mostraba mayores prevalencias que la de Cole.

Flegal ${ }^{(16)}$ también comunicó que, usando la referencia de Must, las prevalencias de obesidad en las niñas y adolescentes era mayor que la dada por Cole; todo lo contrario sucedió con el sobrepeso

Abrantes ${ }^{(17)}$, en niños y adolescentes brasileros, analizó su información com- parando las clasificaciones de Must y Cole, en función de un cuadro $3 \times 3$. Sus resultados fueron similares a los encontrados en el presente estudio, en cuanto a su tendencia se refiere, o sea que usando la de Cole hay mayor prevalencia de sobrepeso y menor de obesidad que con la de Must.

En un estudio en el Brasil, se encontró que la obesidad (por encima del 85 percentil) fue $14,6 \%$ usando la de Cole y $11,8 \%$, con la de Must ${ }^{(18)}$.

En el Perú, en un estudio de 11743 niños de 6 a 12 años, de un nivel socioeconómico alto, se halló las mismas diferencias del presente estudio ${ }^{(19)}$.

Todos los estudios mencionados, independientemente de las prevalencias en sí, reflejan diferencias que siempre son dadas en el mismo sentido. Sin embargo, existen algunos estudios cuyas conclusiones son discordantes a las nuestras. El estudio llevado a cabo en México (20) encontró variaciones en el diagnóstico que iban desde 17 a 39\% de prevalencia de sobrepeso y obesidad, pero a favor de la de Must

Zimmerman ${ }^{(21)}$ estudió la comparación de referencias; en este caso usó la de Cole, con la que recomiendan los Centros para Control y Prevención de la Enfermedad (CDC) y también encontró diferencias; en este caso, quien daba mayores prevalencias fue la del CDC.
No hay que perder de vista que las referencias poblacionales son referencias basadas en definiciones estadísticas y no en estudios que hayan asociado riesgos en la salud con valores elevados de IMC. Por esa razón, se dice que los diagnósticos se basan en criterios estadísticos y no necesariamente se correlacionan con un riesgo biológico ${ }^{(22)}$.

La de Must ha sido criticada por ser una representación exclusivamente estadounidense. Cole también reconoció que la población utilizada en su referencia no es la ideal, por cuanto de alguna manera refleja a poblaciones occidentales y que faltarían algunos otros tipos de poblaciones para compensar esta deficiencia. La de la OMS es considerada la más adecuada, por haber estudiado países que de alguna manera reflejan la diversidad que existe en el mundo, y que la selección de los niños ha sido hecha a través de requisitos que aseguran que tienen una buena calidad de vida. Sin embargo, todas estas cualidades se refieren al grupo preescolar; la presentación de los valores referenciales de IMC de los 6 a los 18 años ha sido construida en base a un modelo matemático, en donde están los preescolares y los otros pertenecen a la población de Must. Cada método tiene sus ventajas y limitaciones y por eso se recomienda que, cuando sean usadas con fines de comparación, se utilice las mismas poblaciones referenciales.

\section{REFERENCIAS BIBLIOGRÁFICAS}

1. Organización Mundial de la Salud (OMS). Medición del cambio del estado nutricional. Ginebra: OMS; 1983.

2. Gómez F. Desnutrición. Bol Med Hosp Inf (México). 1946;3:4

3. National Center for Health Statistics. Growth Charts. Rockville, MD: US Department of Health, Education and Welfare, Public Health Service, Health Resources Administration; 1976.

4. World Health Organization (WHO). Expert Committee, Physical Status: the use and interpretation of anthropometric. Report of a WHO Expert Committee. World Health Organization Tech Rep Ser. 1995;854:1-452.

5. Must A, Dallal G, Dietz W. Reference data for obesity: 85th and 95th percentiles of body mass index (wt/ht2)-a correction. Am J Clin Nutr. 1991;54:773. 
6. Cole T, Bellizi M, Flegal K, Dietz W. Establishing an standard definition for child overweight and obesity worldwide: international survey. BMJ. 2000;320:1240-3.

7. Centers for Disease Control and Prevention [Internet]. CDC growt charts. Atlanta, GA: CDC; 2000 [citado el 22 noviembre de 2008]. Disponible en: http://www.cdc.gov/growthcharts/ 2000

8. De Onis M, Onyango A, Borghi E, Siyam A, Nishida Ch, Siekmann J. Development of a WHO growth reference for school-aged children and adolescents. Bulletin of the World Organization. 2007;85:660-7.

9. Lohman T, Roche A. Anthropometic Standardization Reference Manual. Illinois Champaign: Human Kinects Books; 1990.

10. Ministerio de Salud, Instituto Nacional de Estadística (INE). Encuesta Nacional de Nutrición y Salud (ENNSA 1984). Lima: MINSA, INE; 1984.

11. Ministerio de Salud, Instituto Nacional de Salud, Centro Nacional de Alimentación y Nutrición (CENAN). Monitoreo Nacional de Indicadores Nutricionales (MONIN 2003). Lima: MINSA, INS, CNAN; 2003.

12. Instituto Nacional de Estadística (INE). Encuesta Demográfica y de Salud Familiar (ENDES 2005). Lima: INE; 2005.

13. Pajuelo J, Maraví K. Comparación de dos sistemas de clasificación para el diagnóstico de la obesidad en niños de 2 a 5 años. IX Congreso Peruano de
Endocrinología. Lima, Perú. 2002.

14. Pajuelo J. La obesidad infantil en el Perú. Lima: Facultad de Medicina-UNMSM; 2003.

15. Wang Y, Wang JQ. A comparison of international references for the assessment of child and adolescent overweight and obesity in different populations. Eu J Clin Nutr. 2002;56:973-82.

16. Flegal K, Ogden C, Wei R,Kuczmarski R, Johnson C. Prevalence of overweight in US children: comparison of US growth charts from The Center for Disease Control and Prevention with other reference values for body mass index. Am J Clin Nutr. 2001;73:1086-93.

17.Abrantes M, Lamounier J, Colosimo E. Recommendations for the use of body mass index for the classification of overweight and obese children and adolescents. Food Nutr Bull. 2002;23:262-6.

18. Reiffe Viera A, Marino M, Ramos de Marius V, Sichieri $R$, Valeria de Veiga $G$. Desempenho de pontos de corte do índice de massa corporal de diferentes references nao presicao de gordura corporal en adolescents. Cad Saude Publica. 2006;22(9):1681-90.

19. Pajuelo J. La situación nutricional de niños y adolescentes de un nivel socioeconómico alto de Lima Metropolitana. Lima: Facultad de Medicina UNMSM, Nestlé SA.; 2008.

20. Ramírez E, Grijalva-Haro MI, Ponce J, Ramírez M. Prevalencia de sobrepeso y obesidad en el noroeste de México por tres referencias de Índice de Masa Corporal: diferencias en la clasificación. Archivos Latinoamericanos de Nutrición. 2006;56(3):33-9.

21.Zimmermann M, Gubeli C, Puntener C, Molinari L. Detection of overweight and obesity in a national sample of 6-12-y-old Swiss children: accuracy and validity of reference values for body mass index from the US Centers for Disease Control and Prevention and The International Obesity Task Force. Am J Clin Nutr. 2004;79:838-43.

22. Kain J, Uay R, Vio F, Albala C. Trends in overweight and obesity prevalence in Chilean Children: comparison of three definitions. Eur J Clin Nutr. 2002;56:200-4.

Manuscrito recibido el 17 de agosto de 2009 y aceptado para publicación el 4 de setiembre de 2009.

Correspondencia:

Dr. Jaime Pajuelo Ramírez

Instituto de Investigaciones Clínicas

Hospital Nacional Dos de Mayo

Av. Grau cuadra 13. Parque Historia de la Medicina.

Lima 1, Perú

Correo-e: japara18@yahoo.com 